\title{
Central and peripheral corneal thickness measurement with Orbscan II and topographical ultrasound pachymetry
}

\author{
Jose Manuel González-Méijome, OD, Alejandro Cerviño, OD, Eva Yebra-Pimentel, OD, PhD, \\ Manuel A. Parafita, MD, PhD
}

\begin{abstract}
Purpose: To compare thickness measurements of the central $6.0 \mathrm{~mm}$ of the cornea obtained with the $\operatorname{Orbscan}^{\circledR}$ II topography system and topographical ultrasound pachymetry.

Setting: School of Optometry, University of Santiago de Compostela, Galicia, Spain.

Methods: In 24 right eyes, pachymetric measurements were taken at the center and $1.2 \mathrm{~mm}$ and $3.0 \mathrm{~mm}$ on the superior and inferior hemimeridians. A 1-sample $t$ test was applied to assess the significance of the relationship between Orbscan II and ultrasound methods. The relationship between the 2 was assessed by analyzing regression and plotting the differences against the mean corneal thickness. Orbscan II data were analyzed in 3 ways: (1) without the application of an acoustic equivalent correction factor; (2) with a correction factor of 0.92 , as recommended by the manufacturer; (3) with correction using the equations derived in this study. The data were systematically compared with those of ultrasound pachymetry.
\end{abstract}

Results: Before the correction factor was applied, the Orbscan II overestimated the corneal thickness at all locations, with the mean difference $(48.15 \mu \mathrm{m} \pm$ 33.74 [SD]) significantly different from zero $(P<.001)$. Differences increased toward the periphery, and the reliability of Orbscan II readings seemed to decrease with thicker corneas. After the acoustic equivalent was applied, the differences were significantly less; however, this effect did not seem clinically significant as large differences remained. When specific corrective equations were applied for each corneal location, the level of agreement between Orbscan II and ultrasound pachymetry improved substantially; the mean $(-0.11 \pm 15.22 \mu \mathrm{m})$ was not statistically different from zero $(P>.05)$.

Conclusions: The acoustic equivalent correction factor proposed by the manufacturer to obtain corneal thickness measurements with the Orbscan II compared to those from ultrasound pachymetry was not valid for all corneal topography positions. Orbscan II measurements agreed better with those of ultrasound pachymetry when equations for the central and each peripheral location across the topography were applied.

J Cataract Refract Surg 2003; 29:125-132 @ 2003 ASCRS and ESCRS

$\mathrm{C}$ orneal thickness measured by pachymetry is a sensitive indicator of corneal health. One of the most common approaches to corneal pachymetry is ultrasound technology. Corneal thickness measured by pachymetry is now considered essential in the diagnosis of multiple corneal diseases. Its use can avoid complica- tions that may result from refractive surgery procedures. However, the most commonly used methods of measuring corneal thickness - ultrasound and optical pachymetry-may be adversely influenced by changes in tissue hydration caused by ultrasound speed through the cornea. ${ }^{1,2}$ This can decrease corneal transparency, creating 
a significant handicap to performing accurate measurements with optical pachymetry in some situations. ${ }^{3,4}$ Today, more sophisticated devices are available for measuring central and peripheral corneal thickness. ${ }^{5-10}$

Earlier comparisons of optical and ultrasound corneal thickness measurements studied only central measurements, and there was a slight tendency for ultrasound methods to underestimate corneal thickness. ${ }^{2,11}$ However, ultrasound pachymetry is less affected by error than optical measurement and has the benefit of portability. ${ }^{12,13}$

Both measurement principles have been applied to develop modern devices that map the thickness of the entire cornea. These include high-frequency ultrasound biomicroscopy and Orbscan ${ }^{\circledR}$ topography, which are mainly used for screening and follow-up after refractive surgery procedures. The Orbscan II topography system (Orbtek, Bausch \& Lomb Surgical) uses the lateral displacement of 2 slit beams and a video camera. It takes several images of different corneal sections for 3-dimensional reconstruction of corneal tissue, mapping the anterior and posterior corneal surfaces as well as the full corneal thickness.

A study comparing Orbscan and ultrasound pachymetry found systematic differences in central corneal measurements, with ultrasound readings being 23 to $28 \mu \mathrm{m}$ smaller. ${ }^{10}$ However, that study used the first version of the apparatus, Orbscan I, which did not use the acoustic equivalent to convert the actual measurement of the Orbscan system to values that could be comparable to those obtained with ultrasound pachymetry. Similar results were recently reported when Orb-

Accepted for publication August 21, 2002.

From the Department of Physics, School of Sciences, University of Minho, Braga, Portugal (González-Méijome), and the Departments of Surgery (Cerviño, Parafita) and Applied Physics (Yebra-Pimentel), School of Optics and Optometry, University of Santiago de Compostela, Galicia, Spain.

Presented in part at the 32nd Congress of the European Society of Contact Lenses, Santander, Spain, June 2001.

Supported by MEC (Spain)-PGC under contract PM 98-0225.

None of the authors has a financial or proprietary interest in any material or method mentioned.

Reprint requests to Prof. Dr. Manuel Parafita, Escuela de Óptica y Optometría, Universidad de Santiago de Compostela, 15782 Santiago de Compostela, Galicia, Spain. E-mail: ciparaft@usc.es. scan measurements were compared with optical and ultrasound pachymetric measurements; although the study did not tell which Orbscan version was used, it seems as though it was the first. ${ }^{14}$ In contrast, a study comparing Orbscan II with ultrasound pachymetry found that ultrasound pachymetry was consistently thicker when an acoustic equivalent of 0.92 was used for actual Orbscan II readings. ${ }^{15} \mathrm{~A}$ more recent study showed that Orbscan II and ultrasound central pachymetry were comparable when the acoustic factor was considered. ${ }^{3}$

These controversies must be resolved as inaccurate determination of corneal thickness could lead to serious complications in refractive surgery procedures. Underestimating corneal thickness can lead to the exclusion of patients who may be candidates for primary procedures or enhancements in refractive surgery. Overestimating corneal pachymetry can lead to inadvertent thinning of the stromal bed, increasing the risk of secondary keratectasia. $^{16}$

These comparative studies considered only the central cornea; however, clinical procedures such as refractive surgery affect part of the peripheral cornea. Today, many refractive surgeons rely only on Orbscan pachymetry measurements to determine a patient's suitability for laser in situ keratomileusis.

Although the main objective of the new pachymetric devices is to offer complete corneal-thickness data, the precision and accuracy of peripheral measurements have not been fully investigated. It is also important to know the impact of corneal thickness on Orbscan II and ultrasound pachymetry measurements to detect whether one instrument is biased in favor of thinner corneas or thicker corneas.

Thus, we compared peripheral corneal thickness measurements taken with the Orbscan II with those taken by topographical ultrasound pachymetry.

\section{Patients and Methods}

Twenty-four subjects participated in the study. The 10 men and 14 women ranged in age from 21 to 26 years. Inclusion criteria were no ocular disease, no complaints of ocular irritation, no history of contact lens wear, and no corneal fluorescein staining or anterior segment abnormality on biomicroscopic examination. After the procedures were explained, the subjects signed an informed consent form and were enrolled in the study. 
Because conclusions from the analysis of 1 meridian could be extended to any meridian in the cornea, only vertical measurements where taken to simplify the test for the subjects. Corneal thickness was assessed at 7 locations along the vertical meridian of the right cornea (visual center and $1.0 \mathrm{~mm}, 2.0 \mathrm{~mm}$, and $3.0 \mathrm{~mm}$ superiorly and inferiorly from center) with the Orbscan II and topographical ultrasound pachymetry (Paxis ${ }^{\circledR}$, BVI Vision). After the Orbscan II measurements, the cornea was anesthetized with topical tetracaine solution and topographical ultrasound pachymetry was done. Each result was the mean of 3 consecutive measurements. The ultrasound probe was sterilized and applied as perpendicular as possible to the central cornea. A slitlamp examination was performed to ensure that no corneal damage resulted from the application of the probe. To ensure precision, all measurements were done by the same investigator (J.M.G.M.) and ultrasound measurements were obtained with the aid of a fixation panel. ${ }^{17}$

As corneal thickness measurements taken by ultrasound pachymetry can be adversely affected by fluctuations in tissue hydration, contact lens users were excluded from the study and all measurements were done between 4:00 and 8:00 PM. This is considered the time of day when the eye is most physiologically stable so that hydration, which affects US speed, would be more homogeneous.

For Orbscan II measurements, no acoustic equivalent correction was used. However, a correction factor of 0.92 was applied during a subsequent analysis to allow comparison of the results with those in other studies and to estimate the clinical relevance of the factor.

Data were analyzed using SPSS (version 10.0). The differences between Orbscan II and ultrasound pachymeter measurements were tested for statistical significance using the
Student paired $t$ test and independent-samples $t$ test against zero. The level of significance was $P=.05$. Linear regression was used to quantify the correlation between the 2 methods and to extract a valid equation for deriving accurate values of corneal thickness from the Orbscan II; this analysis was performed separately for the central area and the superior and inferior areas $1.0 \mathrm{~mm}, 2.0 \mathrm{~mm}$, and $3.0 \mathrm{~mm}$ from the center. The differences were plotted against the means to estimate the influence of the measured value on the difference between the methods.

\section{Results}

Table 1 shows the means and standard deviations for the 7 corneal positions before and after the application of the correction value to obtain the acoustic equivalent for the Orbscan II measurement. The mean differences are also shown.

Without applying the acoustic equivalent correction, the Orbscan measurements were systematically higher than ultrasound pachymetry measurements and the differences between the methods were larger the farther from the center of the cornea. When the acoustic equivalent correction was applied, the mean difference decreased between 45 and $55 \mu \mathrm{m}$. In this case, the Orbscan II gave lower values than ultrasound at the center and at $1.0 \mathrm{~mm}$. Although there was no statistically significant difference at $2.0 \mathrm{~mm}$, the Orbscan II had a tendency to overestimate corneal thickness at $3.0 \mathrm{~mm}$.

Table 1. Mean $( \pm S D)$ ultrasound pachymetry values and Orbscan II pachymetry values with and without the manufacturer-recommended correction factor.

\begin{tabular}{|c|c|c|c|c|c|}
\hline \multirow{2}{*}{$\begin{array}{l}\text { Corneal } \\
\text { Location }\end{array}$} & \multicolumn{3}{|c|}{$\begin{array}{l}\text { Mean Corneal Thickness }(\mu \mathrm{m}) \\
\text { Without Factor }\end{array}$} & \multicolumn{2}{|c|}{$\begin{array}{l}\text { Mean Corneal Thickness }(\mu \mathrm{m}) \\
\text { with Factor* }\end{array}$} \\
\hline & US & Orbs & Mean Diff & Orbs & Mean Diff \\
\hline \multicolumn{6}{|l|}{ Superior } \\
\hline $3.0 \mathrm{~mm}$ & $558 \pm 40$ & $683 \pm 49$ & 95 & $629 \pm 46$ & 40 \\
\hline $2.0 \mathrm{~mm}$ & $566 \pm 42$ & $620 \pm 48$ & 54 & $571 \pm 45$ & 4 \\
\hline $1.0 \mathrm{~mm}$ & $551 \pm 38$ & $574 \pm 49$ & 23 & $528 \pm 45$ & -23 \\
\hline Center & $545 \pm 40$ & $560 \pm 48$ & 15 & $515 \pm 45$ & -30 \\
\hline \multicolumn{6}{|l|}{ Inferior } \\
\hline $3.0 \mathrm{~mm}$ & $548 \pm 39$ & $572 \pm 48$ & 24 & $527 \pm 44$ & -21 \\
\hline $2.0 \mathrm{~mm}$ & $562 \pm 43$ & $608 \pm 47$ & 45 & $559 \pm 43$ & -3 \\
\hline $1.0 \mathrm{~mm}$ & $577 \pm 45$ & $659 \pm 47$ & 81 & $606 \pm 43$ & 28 \\
\hline
\end{tabular}

US = ultrasound pachymetry; Orbs = Orbscan II pachymetry; Diff = difference

${ }^{*} 0.92 \times$ Orbscan II thickness 


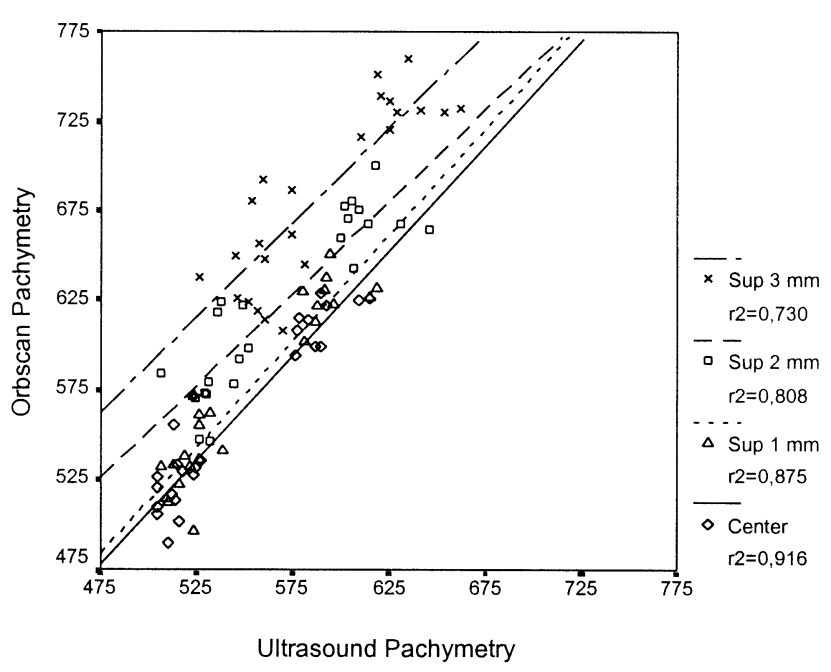

A

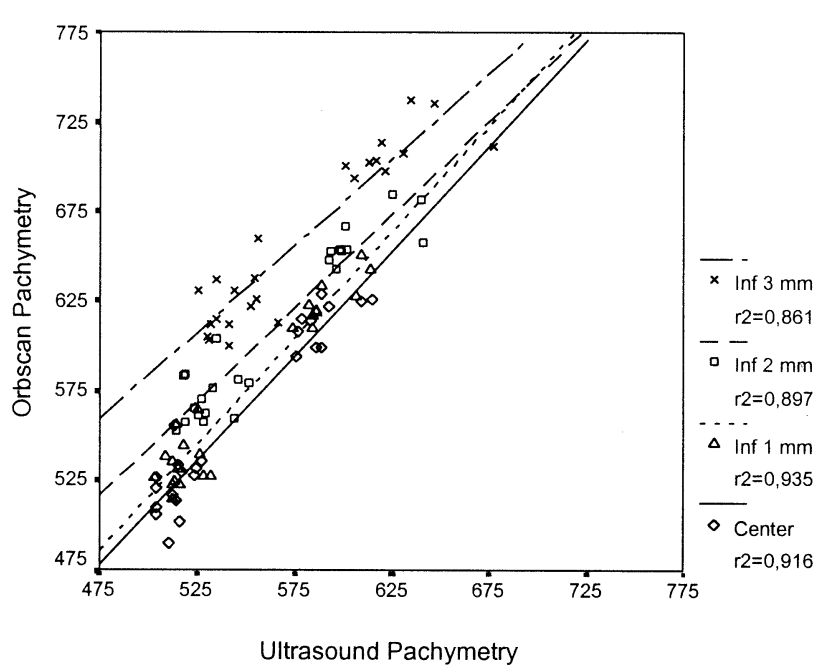

B

Figure 1A. (González-Méijome) Regression lines between ultrasound and Orbscan II pachymetry at superior $(A)$ and inferior $(B)$ locations.

Figure 1A shows the relationship between the 2 methods for superior and inferior measurements before the application of the acoustic equivalent correction factor of 0.92 and Figure 1B, after the application of the factor. The linear regression lines illustrate the results shown in Table 1. As an acoustic equivalent factor was applied to all data, linear regression was numerically equal in terms of the coefficient of determination $\left(r^{2}\right)$, with a different equation for the linear regression line. Thus, the lines approach the 45-degree line, which rep-

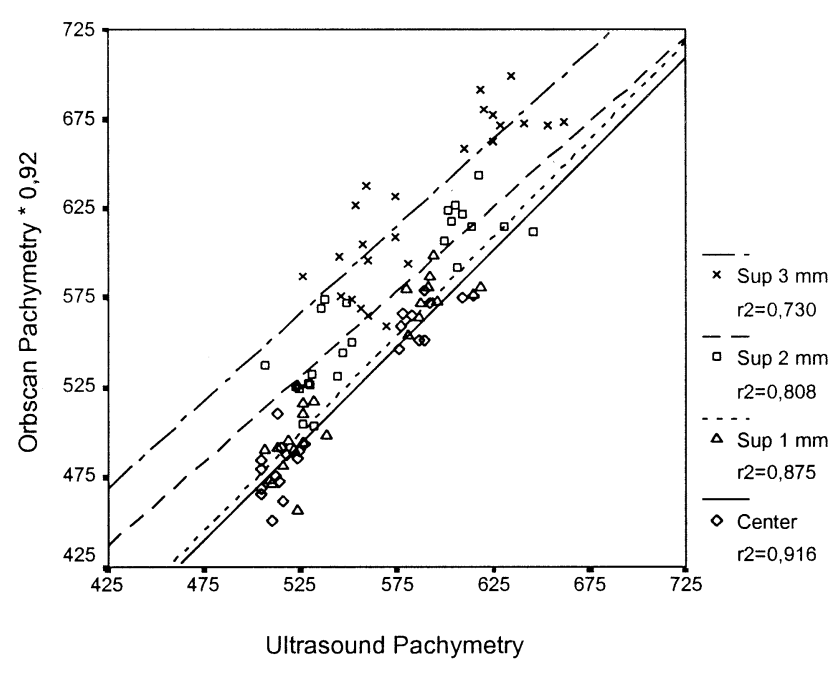

A

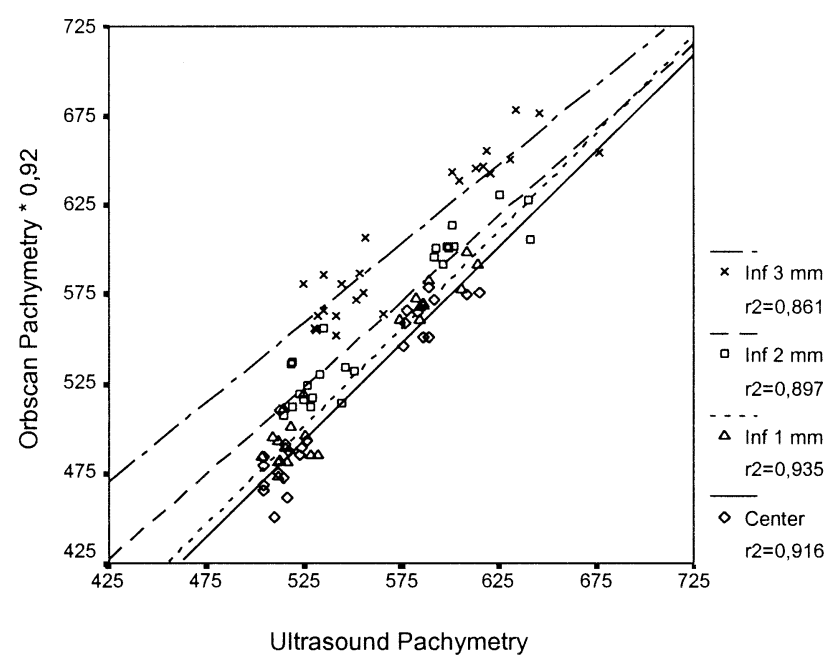

B

Figure 1B. (González-Méijome) Regression lines between ultrasound and Orbscan II pachymetry after the application of an acoustic equivalent correction factor of 0.92 at superior $(A)$ and inferior $(B)$ locations.

resents a perfect correlation between the pachymetric methods. This is particularly evident at the $1.0 \mathrm{~mm}$ inferior position, which shows a better correlation than the central position with a high coefficient of determination $\left(r^{2}=0.935\right)$. Except at the most superior positions, the $r^{2}$ values indicated a good correlation between ultrasound and Orbscan II pachymetry.

Figures $1 \mathrm{~A}$ and $1 \mathrm{~B}$ also show the distribution of the corneal thickness in all 24 eyes. Using ultrasound pachymetry as a reference, closer analysis revealed a 


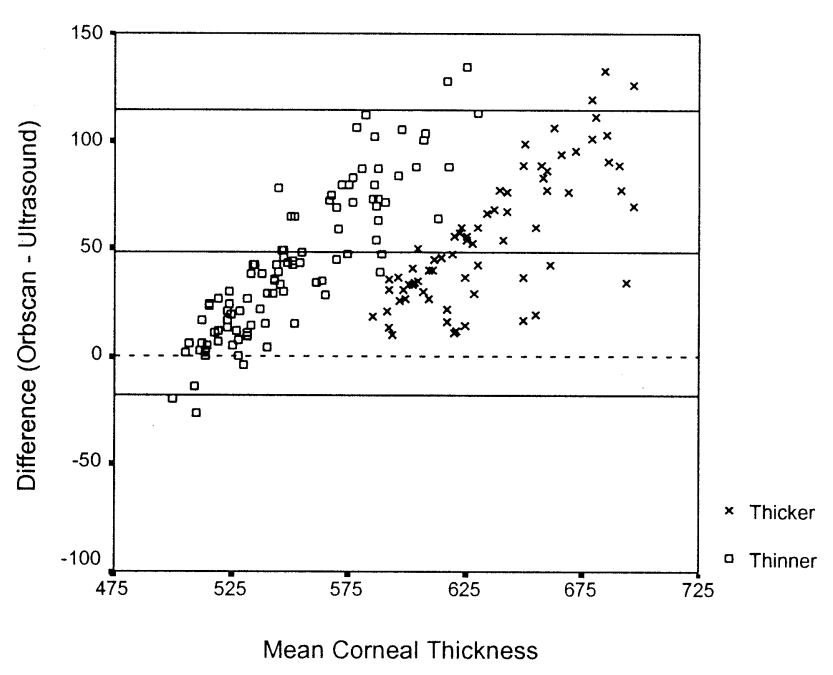

$\boldsymbol{A}$

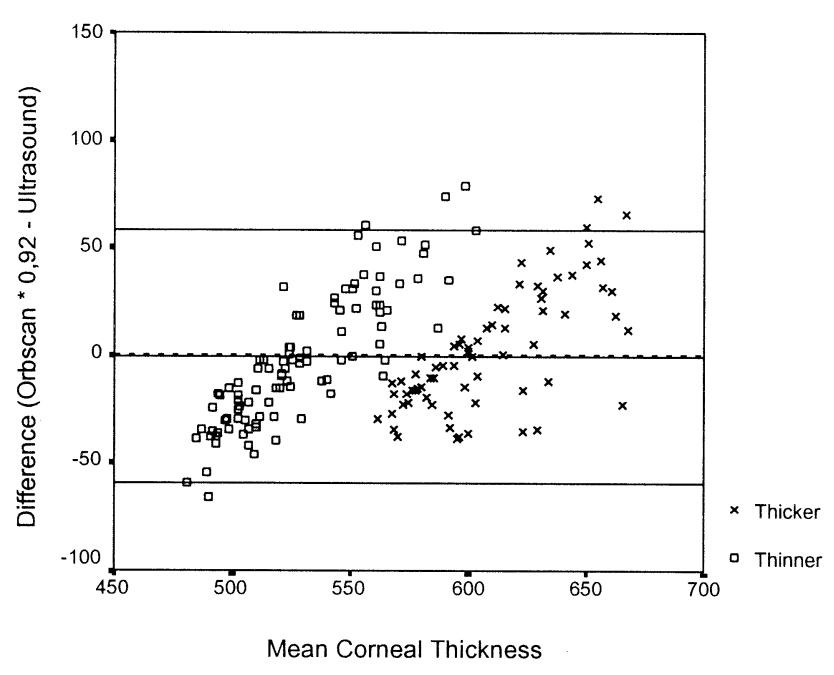

$\boldsymbol{B}$

Figure 2. (González-Méijome) Plots of the differences in corneal thickness between ultrasound pachymetry and Orbscan II before $(A)$ and after $(B)$ the application of the acoustic equivalent correction factor recommended by the manufacturer.

group with central ultrasound pachymetry below $527 \mu \mathrm{m}(\mathrm{n}=14)$ and another with central ultrasound pachymetry over $576 \mu \mathrm{m}(\mathrm{n}=10)$.

Figure 2 shows the difference between methods plotted against the mean values. The thinner and thicker corneas had a different distribution of thickness. Figure 2, $A$ and $B$, shows a comparison of values before and after application of the acoustic equivalent correction to Orbscan measurements, respectively. Most values were within the $95 \%$ limit of agreement between the 2 meth- ods. The mean difference $(48.15 \mu \mathrm{m})$ was significantly different from zero when no acoustic equivalent was considered $(t=18.5 ; P<.001$, independent $t$ test). The mean difference $(-0.72 \mu \mathrm{m})$ was not statistically different from zero when the acoustic equivalent correction factor of 0.92 was considered $(t=-0.31 ; P=$ $.757)$.

The distribution of data changed little after the conversion of Orbscan II values, with a slight displacement of all data around $50 \mu \mathrm{m}$ on the vertical axis, corresponding to the effect of the acoustic equivalent factor of 0.92 , and around $25 \mu \mathrm{m}$ on the horizontal as a result of the average between corrected Orbscan measurements and ultrasound pachymetry. Thus, the $95 \%$ confidence interval (114.28 to -17.98$)$ was less before acoustic equivalent correction than after correction $(-59.23$ to 57.87). There was greater dispersion of data in the group with thicker corneas, which indirectly means that the level of agreement between the 2 pachymetric techniques could depend on the magnitude of thickness to be measured. Table 2 shows a numerical estimation of this effect.

Before applying the corneal factor, the differences between the 2 instruments were greater with thicker corneas (greater error). After the factor was applied, the errors for thicker and thinner corneas become more similar (thickness measured factor becomes less important). Although differences between thinner and thicker corneas for the $2.0 \mathrm{~mm}$ and $3.0 \mathrm{~mm}$ peripheral locations did not change much, the central and $1.0 \mathrm{~mm}$ measurements benefited with the conversion, resulting in fewer differences in thicker corneas.

As bias at different corneal locations was substantially different, a point-to-point analysis of regression was done to obtain expressions that would make data between Orbscan II and ultrasound pachymetry more comparable. Orbscan values II were used as a reference. When no acoustic equivalent was taken into consideration, the analysis provided the equations shown in Table 3. When these equations were applied to original data, a new parameter-the Orbscan ultrasound equivalent-was obtained. The results show a closer correlation between techniques, with a mean thickness of $562.62 \mu \mathrm{m} \pm 40.23$ (SD) for the Orbscan II ultrasound equivalent $(610.89 \pm 64.08 \mu \mathrm{m}$ before correction) and $562.73 \pm 43.08 \mu \mathrm{m}$ for ultrasound pachymetry. Moreover, the mean difference 
Table 2. Mean $( \pm S D)$ ultrasound pachymetry values and Orbscan II pachymetry values with and without the manufacturer-recommended correction factor by corneal thickness.

\begin{tabular}{|c|c|c|c|c|c|c|c|c|c|c|}
\hline \multirow{2}{*}{$\begin{array}{l}\text { Corneal } \\
\text { Location }\end{array}$} & \multicolumn{5}{|c|}{$\begin{array}{l}\text { Corneal Thickness }(\mu \mathrm{m}) \\
\text { of Thinner Corneas* }\end{array}$} & \multicolumn{5}{|c|}{$\begin{array}{l}\text { Corneal Thickness }(\mu \mathrm{m}) \\
\text { of Thicker Corneas }^{\dagger}\end{array}$} \\
\hline & US & Orbs & Diff & Orbs $\times 0.92$ & Diff & US & Orbs & Diff & Orbs $\times 0.92$ & Diff \\
\hline \multicolumn{11}{|l|}{ Superior } \\
\hline $3.0 \mathrm{~mm}$ & $558 \pm 14$ & $647 \pm 27$ & 89 & $595 \pm 25$ & 37 & $632 \pm 16$ & $734 \pm 13$ & 130 & $676 \pm 12$ & 44 \\
\hline $2.0 \mathrm{~mm}$ & $533 \pm 12$ & $584 \pm 24$ & 51 & $537 \pm 23$ & 4 & $613 \pm 15$ & $671 \pm 15$ & 58 & $617 \pm 14$ & 4 \\
\hline $1.0 \mathrm{~mm}$ & $520 \pm 9$ & $537 \pm 21$ & 16 & $494 \pm 19$ & -27 & $594 \pm 13$ & $627 \pm 13$ & 33 & $577 \pm 12$ & -17 \\
\hline Center & $513 \pm 8$ & $522 \pm 17$ & 8 & $480 \pm 15$ & -34 & $589 \pm 13$ & $613 \pm 13$ & 24 & $564 \pm 12$ & -25 \\
\hline \multicolumn{11}{|l|}{ Inferior } \\
\hline $3.0 \mathrm{~mm}$ & $517 \pm 8$ & $534 \pm 13$ & 18 & $492 \pm 12$ & -25 & $591 \pm 13$ & $625 \pm 14$ & 34 & $575 \pm 13$ & -16 \\
\hline $2.0 \mathrm{~mm}$ & $529 \pm 4$ & $570 \pm 14$ & 41 & $525 \pm 13$ & -4 & $609 \pm 19$ & $660 \pm 14$ & 51 & $607 \pm 13$ & -2 \\
\hline $1.0 \mathrm{~mm}$ & $543 \pm 12$ & $622 \pm 16$ & 79 & $572 \pm 15$ & 29 & $626 \pm 22$ & $710 \pm 15$ & 84 & $653 \pm 14$ & 27 \\
\hline
\end{tabular}

US = ultrasound pachymetry; Orbs = Orbscan II pachymetry; Diff = difference

${ }^{*}$ Central corneal thickness by ultrasound $\leq 527 \mu \mathrm{m}$

${ }^{\dagger}$ Central corneal thickness by ultrasound $\geq 576 \mu \mathrm{m}$

Table 3. Regression equations to obtain the ultrasound equivalent of Orbscan II pachymetry (EquUSOrbs) from original Orbscan ॥ measurements with the acoustic equivalent (1) and the mean differences between Orbscan II and ultrasound pachymetry after application of the equations to original Orbscan II measurements.

\begin{tabular}{llcl}
\hline $\begin{array}{l}\text { Corneal } \\
\text { Location }\end{array}$ & \multicolumn{1}{c}{ Equation } & $\begin{array}{c}\text { Mean Difference } \pm \text { SD } \\
\text { (EquUSOrbs }- \text { US) }\end{array}$ & $r^{2}$ \\
\hline Superior & & & \\
$3.0 \mathrm{~mm}$ & EquUSOrbs $=0.69 \times$ Orbs +117.42 & $0.17 \pm 21$ & 0.730 \\
$2.0 \mathrm{~mm}$ & EquUSOrbs $=0.79 \times$ Orbs +78.20 & $1.74 \pm 19$ & 0.808 \\
$1.0 \mathrm{~mm}$ & EquUSOrbs $=0.74 \times$ Orbs +126.51 & $0.42 \pm 14$ & 0.875 \\
Center & EquUSOrbs $=0.78 \times$ Orbs +106.57 & $-1.90 \pm 11$ & 0.916 \\
Inferior & & & $0.84 \pm 10$ \\
$1.0 \mathrm{~mm}$ & EquUSOrbs $=0.79 \times$ Orbs +96.57 & $0.78 \pm 14$ & 0.935 \\
$2.0 \mathrm{~mm}$ & EquUSOrbs $=0.86 \times$ Orbs +40.50 & $-2.82 \pm 17$ & 0.816 \\
$3.0 \mathrm{~mm}$ & EquUSOrbs $=0.89 \times$ Orbs -11.38 & & \\
\hline
\end{tabular}

between the Orbscan II ultrasound equivalent and ultrasound pachymetry $(-0.11 \pm 15.22 \mu \mathrm{m})$ was not significantly different from zero $(t=-0.093 ; P>$ $.05)$. The mean difference before the equations were applied was $48.15 \pm 33.74 \mu \mathrm{m}(P<.001)$. Figure 3 shows the differences after correction plotted against the mean corneal thickness.

\section{Discussion}

Accurate assessment of corneal thickness is important in many clinical situations such as the diagnosis of corneal ectatic conditions and corneal physiology, contact lens research, or refractive surgery procedures. Corneal thickness has been evaluated by various methods, and a wide array of literature on the topic is available. ${ }^{5}$ Ultrasound pachymetry has been the standard for estimating corneal thickness for the past few decades. Now that modern pachymetric techniques are commercially available, ultrasound pachymetry is the reference against which the rest are currently tested. ${ }^{10,14,15,18,19}$

However, even the most current comparisons have focused on the central cornea. To our knowledge, no data on the accuracy of new pachymetric methods at 


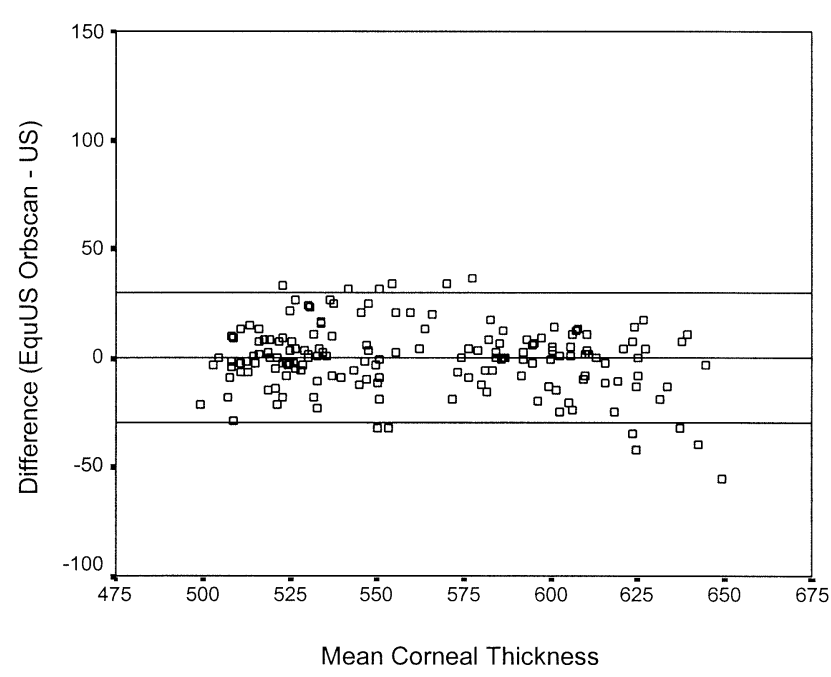

Figure 3. (González-Méijome) A plot of the differences in corneal thickness between ultrasound pachymetry and Orbscan II after the application of the equations listed in Table 3to obtain the ultrasound equivalent of Orbscan II pachymetry (EquUS Orbscan). Note that the scale for the differences is the same as in Figure 2to better show the reduction in bias.

peripheral locations have been published. Of particular interest is the accuracy of peripheral corneal thickness measurements by the Orbscan topography system as this system is widely used by refractive surgeons for important preoperative examination procedures.

Early comparisons with ultrasound pachymetry demonstrated that the Orbscan overestimates central corneal thickness by 23 to $28 \mu \mathrm{m}$, suggesting that this difference could be caused by the absence of tear film after the application of the probe during ultrasound pachymetry. ${ }^{10}$ The Orbscan II theoretically gives more accurate corneal thickness readings by applying an acoustic equivalent to the readings obtained by ultrasound. The value recommended by the manufacturer, 0.92 , is a constant for the entire cornea, which is useful in obtaining comparable central cornea results between the Orbscan II and ultrasound. ${ }^{3}$ However, we found a bias in methods that varies from the center to the periphery; thus, this factor would not correct the disagreement between Orbscan and ultrasound pachymetry values at all points on corneal topography.

Our study, the first comparison of topographical ultrasound pachymetry and Orbscan II to our knowledge, offers 2 key findings. First, when the acoustic equivalent is not applied, bias between techniques leads the Orbscan II to overestimate corneal thickness, with the difference growing toward the periphery. Second, even after the application of the acoustic equivalent, substantial differences remain between the 2 methods. This suggests that sources of error other than tear film are involved, which is why a mean value of $40 \mu \mathrm{m}$ is used as suggested by some of the more recent estimations. ${ }^{20}$

Although the mean differences between Orbscan II and ultrasound pachymetry are significantly less when the equivalent acoustic correction recommended by the manufacturer is considered, this effect does not seem to have much clinical relevance in terms of accuracy. It does not affect the nature of the algorithms applied and induces the same systematic and linear modification of central and peripheral corneal thickness measured by the Orbscan II. Thus, the system underestimates or overestimates corneal thickness as we move toward the periphery of the cornea. For this reason, and on the basis that the same correction factor would not be applied to all points with clinical purposes, we suggest equalizing the acoustic equivalent to a unit. Under this condition, in which no acoustic equivalent is applied, the reliability of the system in terms of concordance with ultrasound pachymetry seems dependent on the thickness of the cornea to be measured. Our results indicate that reliability decreases with thicker corneas. Clinicians would know that corneal pachymetry will be systematically overestimated at all points with the Orbscan II. Until algorithms are improved, correction factors must be specified for each location and probably for each range of measurement.

Moreover, conclusions based on our results cannot be applied with the same level of confidence to corneas having refractive surgery or with corneal ectatic and degenerative dystrophy. In most cases, dystrophy is considered a contraindication to refractive surgery.

We present a first approach to guide clinicians on how to achieve more accurate Orbscan II pachymetry corneal thickness measurements when border values are found. This approach can also be used for diagnosis or in deciding whether to perform refractive surgery in individual cases. The equations we propose reduced bias below $10 \%$ for the central $6.0 \mathrm{~mm}$ of the cornea in all cases analyzed. This implies a substantial reduction from the original Orbscan II data, even when the acoustic equivalent correction factor recommended by the manufacturer is applied. Although measuring the central $6.0 \mathrm{~mm}$ of the cornea is usually enough for 
refractive surgery, it would be beneficial to study the entire cornea.

In summary, the Orbscan II is a noninvasive, rapid scanning system that evaluates corneal thickness across the entire corneal surface. This makes it the most suitable tool to obtain full corneal thickness. Applications in which reproducible measurements from several follow-up visits (precision) are required rather than the exact thickness (accuracy) of the full cornea should not to be affected much by our results; for example, in contact lens research in which physiological alterations of the cornea can be equally detected despite systematic bias in measurement. However, improvements in the algorithms would produce higher accuracy and reliability in procedures that require precise determination of corneal thickness.

When considering refractive surgery, practitioners must pay close attention to the limitations of the system to avoid overestimating corneal thickness, which would increase the risk of keratectasia. Underestimating corneal thickness could lead to excluding patients from having enhancements or primary refractive surgery procedures. It could also lead to false diagnosis of pathological conditions. The reliability of Orbscan measurements can be substantially improved by applying the specific equations we propose to each annular area over the corneal topography.

\section{References}

1. Lattimore MR Jr. Influence of extended soft contact lens wear on the comparative measurement of central corneal thickness. Acta Ophthalmol Scand 1996; 74: 239-242

2. Yebra-Pimentel E, Giráldez MJ, González J, et al. Changes in corneal thickness after daily and extended wear of hydrogel lenses: a comparison of optical and ultrasonic pachometry. Int Contact Lens Clin 1998; 25: 103-107

3. Fakhry MA, Artola A, Belda JI, et al. Comparison of corneal pachymetry using ultrasound and Orbscan II. J Cataract Refract Surg 2002; 28:248-252

4. Reinstein DZ, Aslanides IM, Silverman RH, et al. Highfrequency ultrasound corneal pachymetry in the assessment of corneal scars for therapeutic planning. CLAO J 1994; 20:198-203

5. Parafita M, Yebra-Pimentel E, Giráldez MJ, et al. Optical methods for corneal thickness measurement; a review. In: Pandalai SG, ed, Recent Research Developments in Optics, vol 2. India, Trivandrum Research Signpost, 2002; $35-51$
6. Liu Z, Huang AJ, Pflugfelder SC. Evaluation of corneal thickness and topography in normal eyes using the Orbscan corneal topography system. Br J Ophthalmol 1999; $83: 774-778$

7. Franco S, Almeida JB, Parafita M. Measurement of corneal thickness by videopachymetry: preliminary results. J Refract Surg 2000; 16:S661-S663

8. McLaren JW, Bourne WM. A new video pachometer. Invest Ophthalmol Vis Sci 1999; 40:1593-1598

9. Reinstein DZ, Silverman RH, Raevsky T, et al. Arc-scanning very high-frequency digital ultrasound for $3 \mathrm{D}$ pachymetric mapping of the corneal epithelium and stroma in laser in situ keratomileusis. J Refract Surg 2000; 16:414430

10. Yaylali V, Kaufman SC, Thompson HW. Corneal thickness measurements with the Orbscan Topography System and ultrasonic pachymetry. J Cataract Refract Surg 1997; 23:1345-1350

11. Nissen J, Hjortdal JØ, Ehlers N, et al. A clinical comparison of optical and ultrasonic pachometry. Acta Ophthalmol 1991; 69:659-663

12. Salz JJ, Azen SP, Berstein J, et al. Evaluation and comparison of sources of variability in the measurement of corneal thickness with ultrasonic and optical pachymeters. Ophthalmic Surg 1983; 14:750-754

13. Giasson C, Forthomme D. Comparison of central corneal thickness measurements between optical and ultrasound pachometers. Optom Vis Sci 1992; 69:236-241

14. Marsich MM, Bullimore MA. The repeatability of corneal thickness measures. Cornea 2000; 19:792-795

15. Iskander NG, Penno EA, Peters NT, et al. Accuracy of Orbscan pachymetry measurements and DHG ultrasound pachymetry in primary laser in situ keratomileusis and LASIK enhancement procedures. J Cataract Refract Surg 2001; 27:681-685

16. Geggel HS, Talley AR. Delayed onset keratectasia following laser in situ keratomileusis. J Cataract Refract Surg 1999; 25:582-586

17. Parafita M, Yebra-Pimentel E, Giraldez MJ, et al. Further information on the knowledge of topographical corneal thickness. Int Contact Lens Clin 1999; 26:128137

18. Bovelle R, Kaufman SC, Thompson HW, Hamano H. Corneal thickness measurements with the Topoc SP-2000P specular microscope and an ultrasound pachymeter. Arch Ophthalmol 1999; 117:868-870

19. Wirbelauer C, Scholz C, Hoerauf H, et al. Noncontact corneal pachymetry with slit lamp-adapted optical coherence tomography. Am J Ophthalmol 2002; 133:444450

20. Prydal JI, Artal P, Woon H, Campbell FW. Study of human precorneal tear film thickness and structure using laser interferometry. Invest Ophthalmol Vis Sci 1992; 33:2006-2011 